The effective prompt neutron lifetime in the reactor core was found to be $174 \mu$ sec. with an experimental accuracy of 7 per cent. This is consistent with the accuracy of the delayed neutron data available at present. An improvement in accuracy could be obtained by refinements in the electronic circuit and an improvement in the statistics of the analysis through the use of greater lengths of time-trace records. The variation of the neutron lifetime in different parts of the core can be clearly observed with this technique.

A full report will be published elsewhere.

$$
\text { L. G. Kemeny }
$$$$
\text { SAMAUN }
$$

Nuclear Engineering Laboratory, Queen Mary College, Mile End Road, London, E.1.

1 Moore, M. N., Nucl. Sci. and Eng., 3, 387 (1958).

${ }^{2}$ Barber, N. F., et al., Nature, 158, 329 (1946).

\section{Time-dependent Tensile Strength of Solids}

$\mathrm{HSAIO}^{1}$, considering strain-rate effects on poly. meric and metallic solids, states that for any given value of strain, the stress obtained at different rates of straining is linearly related to the logarithm of the strain-rate. This is considered to be a basic law which is reasonably safe to apply to a large number of solids. $\mathrm{He}$ also presents data for polystyrene showing a linear dependence of fracture stress on the logarithm of strain-rate. These facts are used as evidence supporting certain theories concerning the time-dependence of tensile properties.

The results quoted by Hsaio are confined to a range of two decades of strain-rate in the region of $10^{-5}$ sec. $^{-1}$. In hitherto unpublished work we have measured the tensile properties of isotactic polypropylene (in the form of thin filaments oriented to a draw ratio of about $5 \cdot 5: 1$ ) over six decades of strain-rate from $10^{-4}$ to $10^{3}$ sec. $^{-1}$ at $20^{\circ} \mathrm{C}$. The dependence of stress at a given strain, and of yield and breaking stress, on the logarithm of strain-rate is shown in Fig. 1. These figures reveal that though the conclusions of Hsaio might be applicable to this material in the range of strain-rates in which he worked, they are certainly inapplicable in a range which extends to higher rates of deformation.

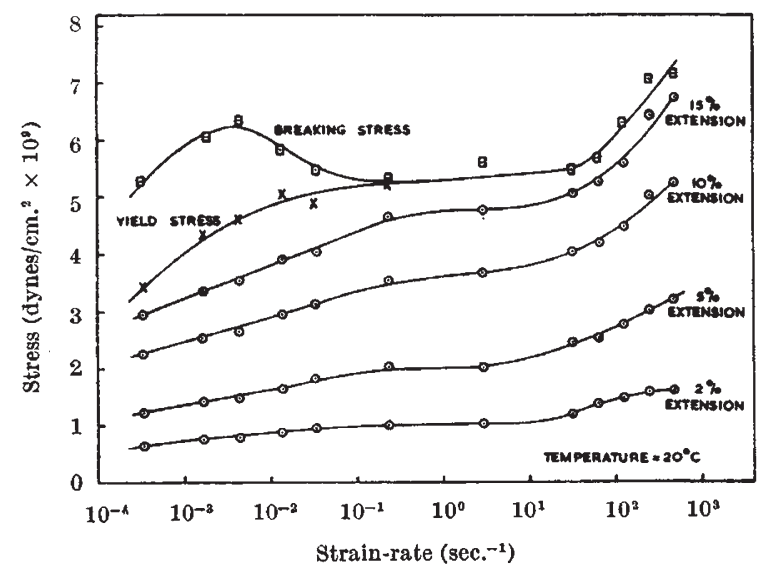

Fig. 1. Effect of strain-rate on tensile properties of oriented isotactic polypropylene
It is intended to publish the full details of this work elsewhere.

I. H. HALI

British Rayon Research Association,

Heald Green Laboratories,

Wythenshawe, Manchester 22.

'Hsaio, C. C., Nature, 186, 535 (1960).

\section{Composition Requirements for Glass Formation in Metallic and Ionic Systems}

WE have developed in recent papers the point of view that all liquids, ionic and metallic included, would form glasses when sufficiently undercooled, save for the intervention of crystallization ${ }^{1-3}$. We have shown ${ }^{2}$ that the glass-forming tendency (for a given rate of cooling and for substances of a given molecular type) is greater the lower is the reduced melting temperature :

$$
\tau_{m}=\frac{k T_{m}}{h_{v}}
$$

where $T_{m}$ is the thermodynamic crystallization temperature, and $h_{v}$ is the molecular heat of vaporiza. tion. This criterion is in semi-quantitative accord with experience on glass formation. In particular it encompasses Zachariasen' ${ }^{4}$ criterion, and accounts for the enhancement of glass-forming tendency by molecular asymmetrization, both being simply equivalent to the depression of $\tau_{m}$. It is also clear, as we pointed out, that the addition of impurities which depress $\tau_{m}$ similarly enhances the glassforming tendency. In this communication we point out striking new experimental confirmation of this last deduction.

Klement, Willens and Duwez ${ }^{5}$ have recently reported the formation of glass in a gold-silicon alloy containing 25 atomic per cent silicon. This appears to have been the first instance in which a metallic glass was formed by cooling the melt. Glass formation in this experiment was, of course, facilitated by the drastic cooling-rate achieved. However, that the gold-silicon alloy in particular formed a glass is, we believe, a consequence of its low $\tau_{m}$. The composition of the alloy is near the eutectic (reported ${ }^{6}$ to be 31 atom per cent silicon) for which $\tau_{m} \sim 0.015$. Thus its $\tau_{m}$ and that of neighbouring compositions are low enough for a strong glass-forming tendency to exist. A similar strong tendency toward glass formation should be exhibited by gold-germanium alloys of near eutectic compositions and indeed by any eutectic with a similar pronounced dip in the melting temperature.

We infer from the reported room temperature instability that the glass transition temperature, $\tau_{g}$ (see ref. 3 for definition), of the gold-silicon alloy is near room temperature, that is, $\tau_{g} \sim 0.007$. This is a much lower glass transition temperature than for other fluid types and is a consequence of the generally higher fluidity of liquid metals.

Another unique feature of the gold-silicon glass is its high stability against crystallization at tempera. tures much above those at which pure amorphous metal films apparently can crystallize. For example, Hilsch $^{7}$ has shown that such films may crystallize at temperatures below $20^{\circ} \mathrm{K} .(\tau \sim 0.001)$. The reason for this stability is that, while in the pure material crystallization requires atom motions of less than one 